\title{
Appropriateness of a Physiotherapic Treatment Protocol for the Axillary Web Syndrome, Applied in a Territorial Rehabilitation Service: Description of a Clinical Case
}

\author{
Luca Collebruscoํ, Antonietta Tufi'1, Elisabetta Brunori² \\ ${ }^{1}$ Rehabilitation Unit, National Health Service of Umbria, Perugia, Italy \\ ${ }^{2}$ The Centro Médico y de Rehabilitación Dr. Rozalén, Madrid, España \\ Email:tdr.colle@tiscali.it
}

How to cite this paper: Collebrusco, L., Tufi, A. and Brunori, E. (2020) Appropriateness of a Physiotherapic Treatment Protocol for the Axillary Web Syndrome, Applied in a Territorial Rehabilitation Service: Description of a Clinical Case. Open Journal of Therapy and Rehabilitation, 8, 53-59. https://doi.org/10.4236/ojtr.2020.83005

Received: March 28, 2020

Accepted: July 25, 2020

Published: July 28, 2020

Copyright $\odot 2020$ by author(s) and Scientific Research Publishing Inc. This work is licensed under the Creative Commons Attribution International License (CC BY 4.0).

http://creativecommons.org/licenses/by/4.0/

\begin{abstract}
The "Axillary web syndrome" (AWS) is an early complication following breast cancer surgery with axillary lymph nodes dissection. Case Report: The patient is a 69-year-old woman, retired ex-employee, with outcome surgery right axillary lymphadenectomy with shoulder pain and functional limitation with diagnosis AWS it where it was recommended the physiotherapy. The patient had improvement in terms of shoulder function and pain and quality of life. Conclusions: The description of the clinical case afflicted with AWS and the treatment protocol applied, highlighted the good performance of the results, notably as concerns patient's quality of life. This result could represent a starting point for creating clinical trials aimed at building appropriate rehabilitation route in territorial rehabilitation services.
\end{abstract}

\section{Keywords}

Axillary Web Syndrome, Manual Therapy, Soft Tissue Mobilization, Exercise Therapy, Case Report

\section{Introduction}

Breast neoplasm is the most common form of cancer among women. Currently in Italy over 37,000 new cases are diagnosed per year [1] [2] 25\% of which women below 50 years of age [3].

Axillary surgical procedures have reported, along with post-operative mor- 
bidity of bleeding, infections, presence of lymphedema and shoulder pain, also the axillary fibrous banding syndrome, better known as axillary web syndrome (AWS).

AWS normally appears between the first and the fifth week after surgery [4] [5] [6] diagnosed on $28.86 \%$ of women [7] [8].

The manifestation AWS is mainly at the level of axillary region at surgical dissection, but it can also extend towards the elbow and most serious cases, up to the column of the thumb [9] [10] with upper limb functional deficits [11].

L'AWS presents as a single cord or multiple thin cords in the subcutaneous tissues of the axilla. The cords frequently result in abduction shoulder pain and limited range of motion.

It presents as a tight subcutaneous cord in the axilla.

It can be associated with later lymphedema in a minority of patients.

L'AWS most frequently becomes symptomatic between 2 and 8 weeks postoperatively but can also develop and recur months to years after surgery.

Physical therapy and exercise can reduce pain and increase range of motion.

Education about and awareness of AWS should be promoted for both patients and providers, and regular assessments for AWS should be initiated by health care providers [12].

\section{Case Report}

The patient is a 69-year-old woman, retired ex-employee, with surgical outcome of right quadrant and right axillary lymphadenectomy because of sentinel lymph node's positivity.

The anamnesis furthermore presents a picture of a pharmacologically controlled hypertension, digestive disorders and a clinical history following a surgery done 20 years ago for cholecystectomy.

The patient is address to the territorial rehabilitation service because of her right shoulder pain, specifically in axillary area and the scapula, limiting most of the normal activities of daily life with the presence of lymphatic vessels' fibrosis at the brachial level, further confirmed from the STAWS questionnaire v.2.1 [13].

During the objective examination, the patient has a functional limitation with an active movement preserved up to $80^{\circ}$ of abduction and $130^{\circ}$ of elevation with the presence of pain in the last degrees of the movement.

The cervical spine has been evaluate and excluded as a factor contributing scapula pain.

Cervical mobility was painless and normal on all its planes of movement as well as the cervical radiculopathy identified through upper quadrant tests [14] was absent, the movement and the strength of the right elbow were normally preserved and pain free.

Hypo mobility from the fourth vertebra (D4) to the seventh vertebra (D7) was present at the anterior-posterior vertebral dorsal segmental test of mobility [15]. 
The description of this clinical case is to create an appropriate physiotherapy protocol to deal with patients having AWS in a rehabilitation service, through an active and passive manual therapy of the upper limb and dorsal cervical junction (MAP) together with self-mobilization exercises to do at home (EA), both established the MAP+EA protocol (MAPEA).

The patient was aware about her clinical condition, as well as had clarifications about her case study.

After agreeing to the publication, she signed a free and informed consent form.

\section{Treatment timeline}

Taking charge of MAPEA treatment in the territorial rehabilitation service lasted two months, where the patient underwent the MAP protocol.

The protocol provided two biweekly sessions lasting 45 minutes, with the addition of EA protocol to execute out twice a day for the duration of 20 minutes for the entire duration of MAP.

The follow up was does at 3 months after the last treatment session.

\section{Therapeutic interventions}

The MAP treatment consisted into passive and active mobilization the shoulder joint in supine position until reaching the pain limit in all its planes.

The stretching passive muscular chains of the upper limb combined with breathing and mobilization of the scapula in lateral decubitus.

The transversal mobilization of soft tissues, around the scar along the direction of the minor mobility without both of pain, burning and itching. Once carried out the work at upper limb level, II and III degree Maitland mobilization [16] of the dorsal cervical junction was realize.

The EA protocol provided for a four exercises of self-mobilization to practise at home (Appendix), after the patient had been trained by the physiotherapist at the end of the first session of the first MAP treatment.

\section{Discussion}

The patient collaborated for the entire duration of the work protocol both in time dedicated to evaluation and MAPEA treatment.

The scales considered is Constant-Murley (C.M.S.) [17] [18] [19]; numerical rating scale (N.R.S.); and EuroqoL (EQ-5D), tool that allows measuring the interviewees health status and their quality of life, because of which it is possible to evaluate the health care provided [20].

The outcome assessment tools were administer before the start of treatment (T0), at the end of the last treatment session (T1) and at follow-up.

The collected data they were analysed and reported in Table 1: in the particular case, there was between $\mathrm{T} 0$ and $\mathrm{T} 1$ improvement in percentage of $43 \%$, N.R.S. scale of 59\%, C.M.S. scale and $81 \%$ in EQ-5D questionnaire, while in follow-up phase of $75 \%$ on the N.R.S scale, of $27 \%$ on C.M.S. and of $23 \%$ on EQ-5D (Figure 1). 
Table 1. Data collection outcomes.

\begin{tabular}{cccccc}
\hline & \multicolumn{5}{c}{ Data Collection } \\
\cline { 2 - 6 } OUTCOME & T0 & T1 & Follow-Up & $\begin{array}{c}\text { Variation } \\
\text { T0 - T1 }\end{array}$ & $\begin{array}{c}\text { Variation } \\
\text { T1 - Follow Up }\end{array}$ \\
\cline { 2 - 6 } N.R.S. & 7 & 4 & 1 & $43 \%$ & $75 \%$ \\
Costant Murley & 24 & 58 & 79 & $59 \%$ & $27 \%$ \\
EQ-5D & 0.124 & 0.656 & 0.848 & $81 \%$ & $23 \%$ \\
\hline
\end{tabular}

Key: N.R.S.: Numerical Rating Scale, EQ-5D: Euroqol.

NUMERICAL RATING SCALE (N.R.S.)

COSTANT MURLEY
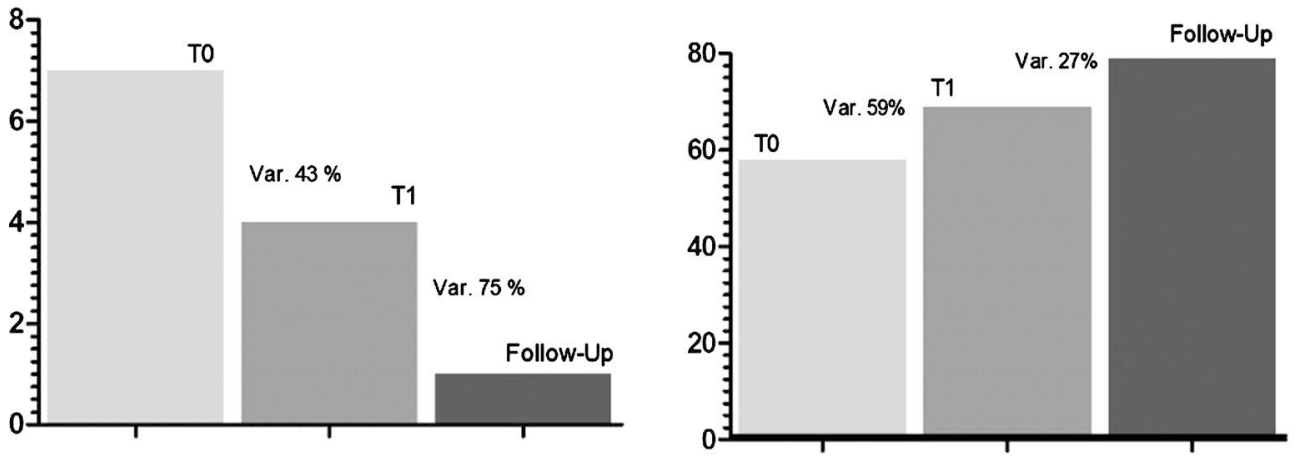

EuroQoL (EQ-5D)

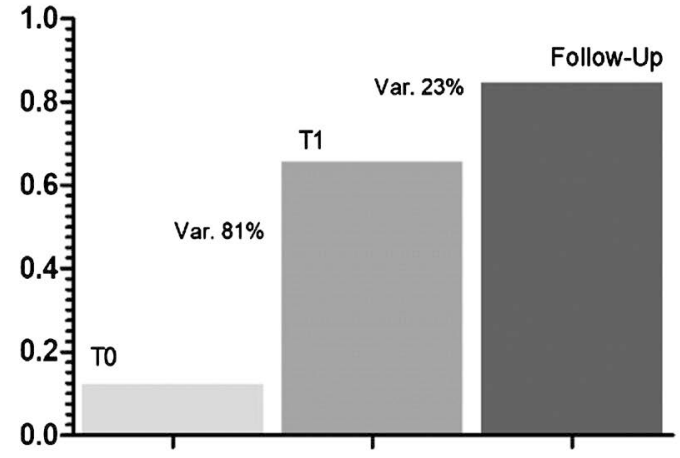

Key: T0: Beginning of treatment, T1: End of treatment, Var.: Variation.

Figure 1. Graphic representation of the score of outcomes, with the percentage change (\%) of improvement between $\mathrm{T} 0$ and $\mathrm{T} 1$ and $\mathrm{T} 1$ and follow-up.

The descriptive analysis has highlighted as the improvement variation at follow-up was did not so high at C.M.S and especially at EQ-5D compared to the outcomes obtained during T1. This result could derive from the fact that the patient was not being treat with MAPEA protocol during the time elapsed between $\mathrm{T} 1$ to follow-up, but she carried out just the EA protocol.

This is a noteworthy aspect and it should be take into account, given that it is widely acknowledged to all rehabilitation health professionals that it is essential having a high level of life's quality in order to keep good levels of rehabilitation assistance. 
The Axillary web syndrome (AWS) is became increasingly recognised as consequence of breast cancer treatment. There are currently no formal guidelines on which to base therapy interventions.

The Physiotherapy intervention for these patients may prove beneficial in limiting subsequent shoulder dysfunction.

This case provides evidence that early physiotherapy intervention may lead to a quicker resolution of symptoms and pain shoulder with improvement quality of life.

The Physiotherapy intervention for these patients may prove beneficial in limiting subsequent shoulder dysfunction. Further research is needed to develop a standardised treatment approach for AWS.

\section{Conclusion}

In literature, there are few works focus attention on the rehabilitation management of the AWS and the clinical case here described could be consider as a tangible model of reference to represent a strategy to further increase works with efficacy tests and range of greater recommendations. The purpose is to device appropriate programs of rehabilitation care in line with what right now appears to be the assistance model for people affected by oncological disease, based increasingly on patient's attention and involvement aimed at improving the quality of life [21].

\section{Acknowledgements}

The authors would like to thank Dr. Ilenia Bardelli for her assistance in giving professional enrichment notions concerning cancer related Lymphedema.

\section{Conflicts of Interest}

The authors declare no conflicts of interest regarding the publication of this paper.

\section{References}

[1] Seigel, R., Naishadham, D. and Jemal, A. (2012) Cancer Statistics 2012. CA: A Cancer Journal for Clinicians, 62, 10-29. https://doi.org/10.3322/caac.20138

[2] Grande, E., Inghelmann, R., Francisci, S., Verdecchia, A., Micheli, A., Baili, P., et al. (2007) Regional Estimates of Breast Cancer Burden in Italy. Tumori Journal, 93, 374-379. https://doi.org/10.1177/030089160709300408

[3] Gangi, S., Coco, V., Di Muro, G., Basile, G. and Basile, F. (2001) Valutazione e trattamento riabilitativo del braccio omolaterale nelle pazienti operate per CA mammario con linfoadenectomia. Europa Medicophysica, 37, 3.

[4] Hack, T., Cohen, L., Katz, J., Robson, L.S. and Goss, P. (1999) Physical and Psychological Morbidity after Axillary Lymph Node Dissection for Breast Cancer. Journal Clinic Oncology, 17, 143-149. https://doi.org/10.1200/JCO.1999.17.1.143

[5] Leidenius, M., et al. (2003) Motion Restriction and Axillary Web Syndrome after Sentinel Node Biopsy and Axillary Clearance in Breast Cancer. American Journal of Surgery, 30, 85-127. https://doi.org/10.1016/S0002-9610(02)01214-X 
[6] Cheville, A.L., et al. (2007) Barriers to Rehabilitation Following Surgery for Primary Breast Cancer. Journal of Surgical Oncology, 18, 95-409. https://doi.org/10.1002/jso.20782

[7] Fukushima, K.F., Carmo, L.A., Borinelle, A.C. and Ferreira, C.W. (2015) Frequency and Associated Factors of Axillary Web Syndrome in Women Who Had Undergone Breast Cancer Surgery: A Transversal and Retrospective Study. SpringerPlus, 4, 112. https://doi.org/10.1186/s40064-015-0889-7

[8] Moskovitz, A.H., Anderson, B.O., Yeung, R.S., Byrd, D.R., Lawton, T.J. and Moe, R.E. (2001) Axillary Web Syndrome after Axillary Dissection. The American Journal Surgery, 181, 434-439. https://doi.org/10.1016/S0002-9610(01)00602-X

[9] Mastrullo, M., Palmieri, M.S., Maestri, A., Puviani, L., Bortone, A., Bellia, R., et al. (2018) Riabilitazione integrata della donna operata al seno. Edra S.p.A, Milano.

[10] Reedijk, M., Boerner, S., Ghazarian, D. and McCready, D. (2006) A Case of Axillary Web Syndrome with Subcutaneous Nodules Following Axillary Surgery. The Breast, 15, 411-413. https://doi.org/10.1016/j.breast.2005.09.005

[11] Veronesi, U. and Luini, A. (1999) Senologia oncologica. Elsevier Masson, Milano.

[12] Koehler, L.A., Haddad, T.C., Hunter, D.W. and Tuttle, T.M. (2019) Axillary Web Syndrome Following Breast Cancer Surgery: Symptoms, Complications, and Management Strategies. Breast Cancer. Targets and Therapy, 11, 13-19. https://doi.org/10.2147/BCTT.S146635

[13] Nevola, L.F., Veronesi, P., Lohsiriwat, V., Luini, A., Schorr, M.C., Garusi, C., et al. (2014) Axillary Web Syndrome Self Assessment Questionnaire: Initial Development and Validation. The Breast, 23, 836-843. https://doi.org/10.1016/j.breast.2014.09.001

[14] Wainner, R.S., Irrgang, J.J., Boninger, M.L., Delitto, A. and Allison, S. (2003) Reliability and Diagnostic Accuracy of the Clinical Examination and Patient Self-Report Measures for Cervical Radiculopathy. Spine, 28, 52-62. https://doi.org/10.1097/00007632-200301010-00014

[15] Flynn, T.W., Cleland, J.A. and Whitman, J.M. (2008) Users' Guide to the Musculoskeletal Examination: Fundamentals for the Evidence-Based Clinician. United States of America: Evidence in Motion.

[16] Maitland, G.D. (1986) Vertebral Manipulation. Butterworth-Heinemann, Boston.

[17] Downie, W.W., Leatham, P.A, Rhind, V.M., Wright, V. and Branco, J.A. (1978) Studies with Pain Rating Scales. Annals of the Rheumatic Disease, 37, 378-381. https://doi.org/10.1136/ard.37.4.378

[18] Grossi, E., Borghi, C., Cerchiari, E.L., Della Puppa, T. and Francucci, B. (1983) Analogue Chromatic Continuous Scale (ACCS): A New Method for Pain Assessment. Clinical and Experimental Rheumatology, 1, 337-340.

[19] Conboy, V.B., et al. (1996) An Evaluation of the Constant-Murley Shoulder Assessment. The Journal of Bone \& Joint Surgery, 78-B, 229-232. https://doi.org/10.1302/0301-620X.78B2.0780229

[20] Rabin, R. and De Charro, F. (2001) EQ-5D: A Measure of Health Status from the EuroQol Group. The Finnish Medical Society Duodecim. Annals of Medicine, 33, 337-343. https://doi.org/10.3109/07853890109002087

[21] Ministero della sanità (2011) Quaderni del ministero della salute: La centralità della Persona in riabilitazione: Nuovi modelli organizzativi e gestionali. http://www.salute.gov.it 


\section{Appendix: Self-Treatment Exercises Protocol, "EA"}
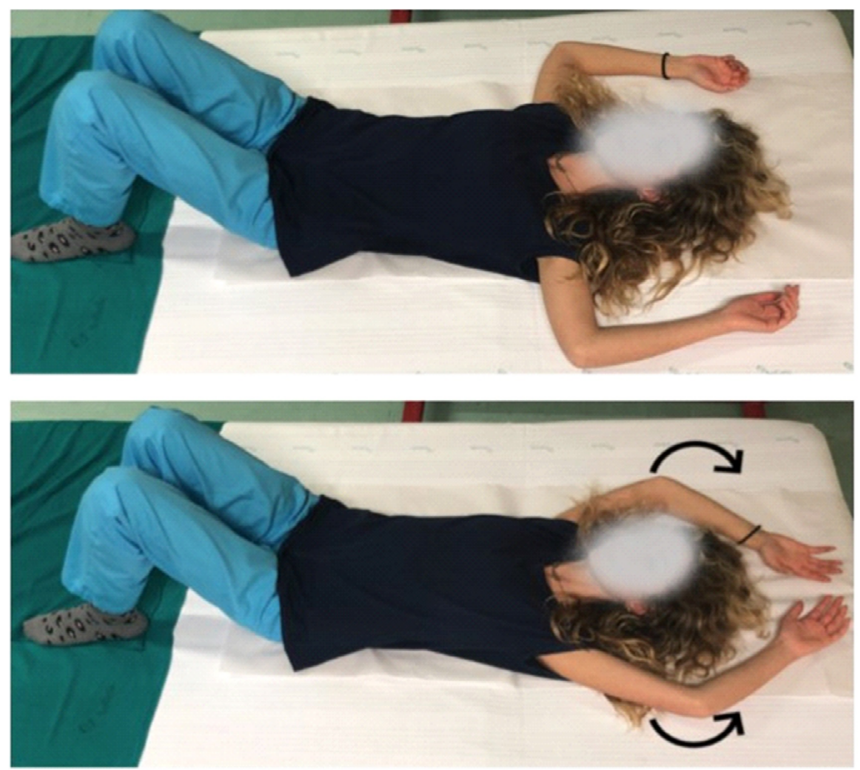

EA 1: In a supine position with abducted arms and elbows flexed at $90^{\circ}$. The patient has to flex her shoulders bringing the hands over her head by sliding the upper limbs over the couch.

Dosage: 3 Repetition $\times$ 6-8 times $\times$ twice a day
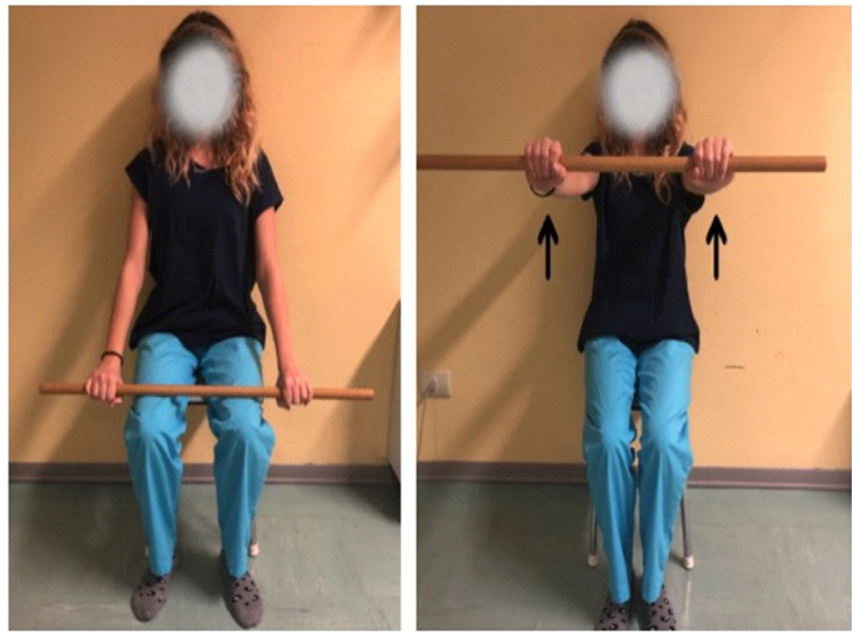

EA 3: Patient sitting, with hands slightly wider than shoulders, the patient holds the cane on her thighs. The movement to be out starting from thighs and has to arrive at $90^{\circ}$ of shoulder flexion.

Dosage: 3 Repetition x 6-8 times $x$ twice a day
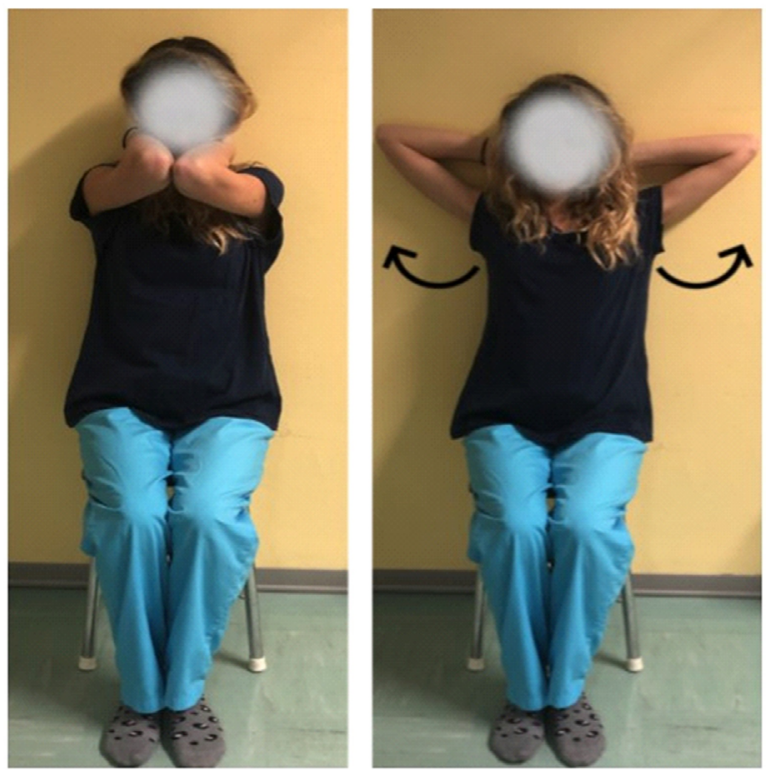

EA 2: Patient sitting with shoulders flexed at $90^{\circ}$, elbows closed to each other and hands behind the nape. The patient has to do an abduction movement on the horizontal plane until she touches the wall.

Dosage: 3 Repetition x 6-8 times $x$ twice a day
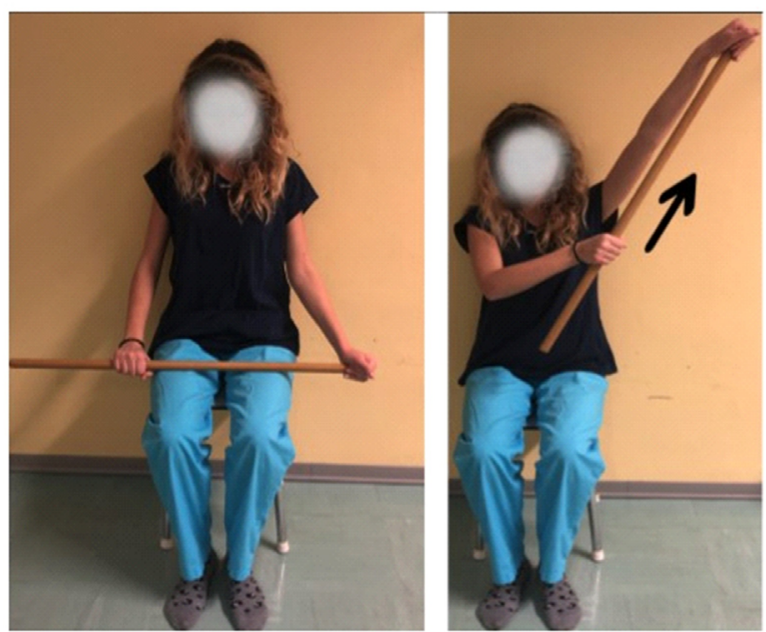

EA 4: Patient sitting, one hand holds the cane at the end and the other one at the half. The hand at the half pushes ward outwards, bringing the other upper limb into abduction and flexion

Dosage: 3 Repetition $\times$ 6-8 times $\times$ twice a day 